\title{
New models for a triaxial Milky Way spheroid and effect on the microlensing optical depth to the Large Magellanic Cloud
}

\author{
C Savage ${ }^{1}$, H J Newberg ${ }^{2}$, K Freese $^{1}$ and P Gondolo ${ }^{3}$ \\ ${ }^{1}$ Michigan Center for Theoretical Physics, Department of Physics, University of \\ Michigan, Ann Arbor, MI 48109, USA \\ 2 Department of Physics, Applied Physics and Astronomy, Rensselaer \\ Polytechnic Institute, Troy, NY 12180, USA \\ ${ }^{3}$ Physics Department, University of Utah, Salt Lake City, UT 84112, USA \\ E-mail: cmsavage@umich.edu, heidi@rpi.edu, ktfreese@umich.edu and \\ paolo@physics.utah.edu
}

Received 23 January 2006

Accepted 15 June 2006

Published 12 July 2006

Online at stacks.iop.org $/ \mathrm{JCAP} / 2006 / \mathrm{i}=07 / \mathrm{a}=003$

doi:10.1088/1475-7516/2006/07/003

\begin{abstract}
We obtain models for a triaxial Milky Way spheroid based on data by Newberg and Yanny. The best fits to the data occur for a spheroid centre that is shifted by $3 \mathrm{kpc}$ from the Galactic Centre. We investigate effects of the triaxiality on the microlensing optical depth to the Large Magellanic Cloud (LMC). The optical depth can be used to ascertain the number of massive compact halo objects (MACHOs); a larger spheroid contribution would imply fewer halo MACHOs. On the one hand, the triaxiality gives rise to more spheroid mass along the line of sight between us and the LMC and thus a larger optical depth. However, shifting the spheroid centre leads to an effect that goes in the other direction: the best fit to the spheroid centre is away from the line of sight to the LMC. As a consequence, these two effects tend to cancel so that the change in optical depth due to the Newberg/Yanny triaxial halo is at most $50 \%$. After subtracting the spheroid contribution in the four models that we consider, the MACHO contribution (central value) to the mass of the Galactic Halo varies from $\sim(8-20) \%$ if all excess lensing events observed by the MACHO Collaboration are assumed to be due to MACHOs. Here the maximum is due to the original MACHO Collaboration results and the minimum is consistent with $0 \%$ at the $1 \sigma$ error level in the data.
\end{abstract}


Keywords: dark matter, lensing, structure of galaxies

ArXiv ePrint: astro-ph/0511046

\section{Contents}

1. Introduction 2

2. Optical depth 3

3. Spheroid models 4

3.1. Star counts and high velocity stars: 'MACHO model' . . . . . . . . . . 5

3.2. Galactic dynamics: 'OC1 model' . . . . . . . . . . . . . . . . . . . 6

3.3. $F / G$ turnoff star counts: 'power law models' and 'Hernquist models' . . . . 6

3.3.1. Position. . . . . . . . . . . . . . . . 7

3.3.2. Triaxiality. . . . . . . . . . . . . . . . 8

3.3.3. Density normalization. . . . . . . . . . . . . . . 9

4. Importance of various factors for optical depth 11

4.1. Triaxiality . . . . . . . . . . . . . . . . . 11

4.2. Position . . . . . . . . . . . . . . . . . 12

4.3. Density profile . . . . . . . . . . . . . . . . . 14

4.4. Density normalization ........................ 15

5. Results 17

5.1. MACHO model . . . . . . . . . . . . . . . . . . . . 17

5.2. Dynamical models $(\mathrm{OC} 1) \ldots \ldots \ldots \ldots$

5.3. New spheroid model: Hernquist profile with Newberg/Yanny fits . . . . . . 18

5.4. Power law model fit to Newberg/Yanny data . . . . . . . . . . . . . . . . . 19

6. Discussion 19

6.1. Triaxiality . . . . . . . . . . . . . . . . . . . . 19

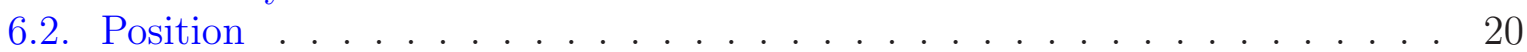

6.3. Density profile. . . . . . . . . . . . . . . . 20

6.4. Normalization . . . . . . . . . . . . . . . . . . . 20

7. Summary 21

Acknowledgments $\quad 22$

References $\quad 22$

\section{Introduction}

The mass of galaxies, including our Milky Way, is composed primarily of unknown dark matter. While the dark matter may consist primarily of elementary particles (such as axions or weakly interacting massive particles), current data indicate that up to $20 \%$ of it may be composed of massive compact halo objects (MACHOs). From a combination of theoretical arguments and data, low mass stars and brown dwarfs have been shown to make 
up no more than a few per cent of the Halo [1]-[7]. Viable MACHO candidates include primordial black holes or white dwarfs. (Constraints on white dwarfs as explanations for MACHO events have previously been discussed in [7]-[9].)

As noted by Paczyński [10], these MACHOs may be detected through gravitational microlensing. A survey of a large number of stars for microlensing events can indicate an excess optical depth due to the MACHOs. Experiments such as MACHO [4], EROS [5, 6], OGLE [11,12], AGAPE [13,14], MOA [15,16], MEGA [17], and Baltz et al [18] have carried out searches for MACHOs by searching for microlensing events in large populations of stars such as the Large Magellanic Cloud (LMC), Small Magellanic Cloud (SMC), Galactic Bulge, M31, and M87. Lensing events have been detected toward the LMC in greater numbers than would be expected for the assumed stellar populations, indicating the possible presence of MACHOs. These experiments have found that as much as $20 \%$ of the Halo of the Milky Way may be composed of a MACHO component.

However, the interpretation of these microlensing results as a new MACHO component requires one to subtract the contribution from known stellar populations. The stellar contribution in turn relies upon the details of the galactic structure - an incorrect model for the stellar distribution may yield an underestimate for this contribution and, hence, lead to some lensing events being mistakenly attributed to MACHOs. One galactic component contributing to the LMC optical depth is the spheroid, or stellar halo, a (roughly spherical) distribution of older stars with a density that falls off more rapidly than the dark halo. The traditional assumption, based upon star counts, is that this spheroid is light and has either a spherical or axial (flattened) shape; the traditional spheroid contributes little to the LMC optical depth. A heavy spheroid has also been considered in the past [19].

Prior to this work, there have been two quite divergent results for the optical depth due to the spheroid. The MACHO experiment used a spheroid optical depth that is one40th of the total optical depth to the LMC. Another model, the OC1 result discussed below, found a spheroid optical depth that is one-third of the total to the LMC.

In this paper we will investigate the consequences of a triaxial shape for the Milky Way spheroid. Newberg and Yanny [20,21] have used $F / G$ turnoff star counts to find a triaxial spheroid. In this paper, we will obtain models for the spheroid based on the Newberg/Yanny data. We will also examine how triaxiality affects the optical depth and determine if triaxial models can account for the excess optical depth toward the LMC.

The paper is organized as follows. In section 2, we will review the determination of the optical depth to the LMC. In section 3, we will present two existing models of the spheroid as well as new triaxial models based upon $F / G$ turnoff star counts. The importance of various factors (the triaxiality, position of the spheroid, and shape and normalization of the density profile) to the optical depth is discussed in section 4. Results are presented in section 5 , discussed in section 6 , and summarized in section 7 .

\section{Optical depth}

In microlensing experiments, a telescope on Earth monitors millions of stars in a nearby galaxy, e.g. the LMC. Occasionally one of these stars will be lensed by an intervening mass, e.g. a MACHO or a star in the spheroid. The distant source star will look brighter during the time period that the intervening mass passes across the line of sight between 
the telescope and the source star. The optical depth is roughly the probability of finding that the source star is lensed. More precisely, in the low optical depth limit that applies to observations of the Milky Way and other nearby galaxies, the optical depth is the probability that a source object (e.g. a star in the LMC) will have its intensity amplified by more than a factor of 1.34 due to gravitational microlensing by an intervening object. It has been shown (see the reviews of Paczynski [22] and Roulet and Mollerach [23]) that the optical depth $\tau$ is

$$
\tau=\frac{4 \pi G}{c^{2}} D_{\mathrm{s}}^{2} \int_{0}^{1} \mathrm{~d} x x(1-x) \rho\left[(1-x) \overrightarrow{\mathbf{r}}_{\odot}+x \overrightarrow{\mathbf{r}}_{\mathrm{s}}\right] .
$$

Here $\overrightarrow{\mathbf{r}}_{\mathrm{s}}$ is the location of the source star, $\overrightarrow{\mathbf{r}}_{\odot}$ is the location of the Sun (we will approximate our position as being at the Sun), and $\rho(\overrightarrow{\mathbf{r}})$ is the mass density of lensing objects at a position $\overrightarrow{\mathbf{r}}$. As density profiles discussed here depend on the radial distance from the centre of the spheroid (traditionally the same as the Galactic Centre), we will choose the origin of the coordinate system to lie at the centre of the spheroid. The coordinate $x$ measures the fractional distance along the line of sight from the Sun toward the source star, where $D_{\mathrm{s}}$ is the distance between us and the source object.

Typically lenses can be expected to traverse the line of sight to source stars, resulting in temporary amplification. The optical depth toward the LMC can then be determined by examining a large number of LMC stars; the average proportion of stars lensed at any one time is the optical depth. Lensing objects with masses $\sim\left(10^{-6}-10^{2}\right) M_{\odot}$ have event timescales on the order of days or months and are observable by the current experiments. The LMC, located at $\left(d_{\mathrm{LMC}}, b_{\mathrm{LMC}}, \ell_{\mathrm{LMC}}\right)=\left(50 \mathrm{kpc},-32.8^{\circ}, 281^{\circ}\right)$, provides a nearby source of stars with a line of sight off the galactic plane that does not pass through the Galactic Centre (the advantage of this line of sight is that it avoids the densest stellar regions, which would overpower the MACHO signal of interest). Several stellar populations will contribute to the optical depth to LMC source stars: the Milky Way disk (both thin and thick components), the Milky Way spheroid, and the LMC disk itself. MACHOs in the Milky Way and LMC haloes will also contribute to the optical depth.

The MACHO Collaboration has determined an optical depth to the LMC of $\tau_{2}^{400}=$ $1.1_{-0.3}^{+0.4} \times 10^{-7}$ (criterion set A, 13 events) and $\tau_{2}^{400}=1.3_{-0.3}^{+0.4} \times 10^{-7}$ (criterion set $\mathrm{B}, 17$ events) [4]; these values of the optical depth are for events with durations between 2 and 400 days. Their prediction for 'known' stellar populations (MW disk, MW spheroid, $\mathrm{LMC}$ disk $)$ is $\tau_{\text {stars }}=(0.24-0.36) \times 10^{-7}\left(0.020 \times 10^{-7}\right.$ from the spheroid $)$, only a fraction of the observed value. The optical depth has a noticeable excess of $\sim 1 \times 10^{-7}$ from the known contributions, with three possibilities (or combination thereof) for this discrepancy: (1) MACHOs, which can contribute up to $20 \%$ of the dark matter halo; (2) backgrounds, such as variable stars, misinterpreted as lensing events; and (3) incorrect stellar population models, yielding 'known' contributions smaller than the actual stellar contributions. Our examination here involves the third case: we will investigate whether a triaxial spheroid will have a larger contribution than the spherical models and account for part of this excess.

\section{Spheroid models}

Modelling the spheroid has presented some difficulty. The spheroid density is expected to fall off faster than the dark halo, with a power law dependence far away from the 
Galactic Centre roughly $\propto r^{-n}$ with $n \sim 3$. However, the inner galaxy profile and total mass (alternatively, local density) are not as well known. Star counts, including surveys of local high velocity stars, and dynamical models based upon rotation curves lead to very different spheroid masses. In this section, we will present a model for each of these cases. In addition, we will examine new models due to Newberg and Yanny [20,21], based upon $F / G$ turnoff star counts.

\subsection{Star counts and high velocity stars: 'MACHO model'}

In principle, the profile of the spheroid could be determined if all of its stars (and other composing objects) could be directly observed. In practice, this becomes difficult as these objects may be too faint to see and may be difficult to distinguish from other stellar populations - the local spheroid density is much smaller $(\sim 0.1 \%)$ than that of the disk. Models can be constructed by observing a distinguishable population of spheroid stars and extrapolating to a total density by using the mass function (MF) [1,2], [24]-[27]. Spheroid stars can be distinguished due to their large relative velocity (disk stars tend to have a small peculiar velocity as they rotate about the centre of the galaxy along with the Sun) or their chemical composition; star counts at high galactic latitudes (away from the disk) can also be used [27]-[39].

One assumption made in these types of determination is an extrapolation of stellar mass functions to the unobservable low mass end of the spectrum (e.g. brown dwarfs); typical extrapolations predict only a small portion of the mass density is composed of brown dwarfs. Atypical models that propose larger numbers of brown dwarfs are ruled out by a combination of theoretical arguments and data [1]-[7]. In particular, the timescales of observed MACHO events are inconsistent with large numbers of very light or very heavy objects, requiring mainly objects of comparable mass to main sequence stars.

The MACHO Collaboration uses a $r^{-3.5}$ power law model based partly upon the kinematically selected observations [4],

$$
\rho(r)=\rho_{\mathrm{S} 0}\left(\frac{r}{R_{0}}\right)^{-3.5} \quad(\mathrm{MACHO})
$$

with a local spheroid density (in the solar vicinity) of

$$
\rho_{\mathrm{S} 0}=1.18 \times 10^{-4} M_{\odot} \mathrm{pc}^{-3} .
$$

Here $R_{0}$ is the distance between the Sun and the Galactic Centre. We will refer to this power law fit as the MACHO profile. Due to the statistical and systematic errors and assumptions made in the determination of this local density used by the MACHO Collaboration, it is possible that it is off by at most a factor of two [40].

Other observations further constrain the local density. The kinematically selected observations lead to estimates of the local spheroid density of $\rho_{\mathrm{S} 0} \approx(1-3) \times 10^{-4} M_{\odot} \mathrm{pc}^{-3}$, where these values may contain significant statistical and systematic errors. The high galactic latitude results of Gould et al [27] yield a noticeably smaller estimate of $6.4 \times 10^{-5} M_{\odot} \mathrm{pc}^{-3}$; the latter analysis relies upon an extrapolation from distant to nearby densities that depends on the spheroid model and could be an underestimate. A recent paper by Gould [39], with a large number of kinematically selected stars, presents a luminosity function from which a more precise value of the local density might possibly 
be obtained. However, systematics in the data and errors in extrapolating the luminosity function to a local density must then be considered; this analysis has not yet been done to our knowledge. Even with all the uncertainties in all these measurements taken into account, the values of the local densities used by the MACHO group or obtained by these other measurements are still in severe disagreement with the local density used in the OC1 model described in the next section: $\mathrm{OC} 1$ gives a value of the local density that is an order of magnitude higher than that used by the MACHO group. Later, we will also examine new work based upon $F / G$ turnoff star counts [20,21], where the estimates of the local density are lower.

However, it may not be appropriate to compare the local density, as measured by counting stars in the solar neighbourhood, with the local stellar density of stars calculated from the Newberg/Yanny triaxial spheroid. If the spheroid is lumpy or contains multiple components, then the local density could be significantly higher than interpolated from the triaxial spheroid, which was fitted to a selection of halo stars which avoided known halo substructures. In section 3.3, we calculate the contribution to the local density expected from each of four best fit $F / G$ spheroid models. In section 4.4, we further discuss the local density of all the spheroid models.

\subsection{Galactic dynamics: 'OC1 model'}

Alternatively, models can be generated based upon dynamical measurements, using simulations to match observed rotation curves [41]-[43]. Such models tend to lead to much heavier spheroids than those based upon star counts, by as much as a factor of 10 . Giudice et al [19] examined several different models for the spheroid; here, we will use the one they refer to as OC1 (proposed by Ostriker and Caldwell [42]). This model is based upon a variety of observational data, but depends significantly upon rotation curves for $R<R_{0}$ as determined from infrared surveys. For the radii relevant to the LMC line of sight,

$$
\rho(r)=\rho_{\mathrm{S} 0}\left(\frac{r}{R_{0}}\right)^{-3}
$$

with a local spheroid density of $\rho_{\mathrm{S} 0}=1.28 \times 10^{-3} M_{\odot} \mathrm{pc}^{-3}$, an order of magnitude larger than that used by MACHO. We note that this 'local density' is obtained from the modelling, which is designed to match a number of data sets, rather than directly from local observations.

\section{3. $F / G$ turnoff star counts: 'power law models' and 'Hernquist models'}

Recently, Newberg and Yanny $[20,21]$ have provided new models for the spheroid by examining $F / G$ turnoff stars using the Sloan Digital Sky Survey (SDSS) $[44,45]$. Models are constructed by taking the SDSS data, removing regions of known clumps of stars and contamination (including streams and other remnants), and assuming a smooth distribution for the remaining regions. The number density of spheroid $F / G$ turnoff stars was fitted to a modified Hernquist profile [46]:

$$
n_{F / G}(r)=\frac{C}{r^{\alpha}(a+r)^{\delta}},
$$


Table 1. Several fits of $F / G$ turnoff star counts to the modified Hernquist profile (equation (5)). Parameters denoted by $(\dagger)$ have been fixed; all other parameters are permitted to vary. Models $\mathrm{H} 1$ and $\mathrm{H} 2$ have true Hernquist profiles, while P1 and $\mathrm{P} 2$ have power law profiles. $\mathrm{H} 1$ and $\mathrm{P} 1$ have the spheroid centre fixed to lie at the Galactic Centre; the centre is free to move in H2 and P2. The 14 fit parameters are described in the text.

\begin{tabular}{|c|c|c|c|c|c|}
\hline Model & & H1 & $\mathrm{H} 2$ & $\mathrm{P} 1$ & $\mathrm{P} 2$ \\
\hline Magnitude & $M_{f}$ & $4.2^{\dagger}$ & $4.2^{\dagger}$ & $4.2^{\dagger}$ & $4.2^{\dagger}$ \\
\hline \multirow[t]{4}{*}{ Location } & $R_{0}(\mathrm{kpc})$ & 8.5 & $8.0^{\dagger}$ & 8.5 & $8.0^{\dagger}$ \\
\hline & $\Delta x(\mathrm{kpc})$ & $\mathbf{0}^{\dagger}$ & 0.1 & $0^{\dagger}$ & 0.2 \\
\hline & $\Delta y(\mathrm{kpc})$ & $0^{\dagger}$ & 3.5 & $0^{\dagger}$ & 2.9 \\
\hline & $\Delta z(\mathrm{kpc})$ & $0^{\dagger}$ & 0.1 & $0^{\dagger}$ & 0.0 \\
\hline \multirow[t]{4}{*}{ Profile } & $\alpha$ & $\mathbf{1}^{\dagger}$ & $\mathbf{1}^{\dagger}$ & 3.0 & 2.9 \\
\hline & $\delta$ & $3^{\dagger}$ & $3^{\dagger}$ & $0^{\dagger}$ & $0^{\dagger}$ \\
\hline & $a(\mathrm{kpc})$ & 14 & 15 & - & - \\
\hline & $C\left(\mathrm{kpc}^{(\alpha+\delta-3)}\right)$ & $2.09 \times 10^{8}$ & $1.55 \times 10^{8}$ & $2.16 \times 10^{6}$ & $1.07 \times 10^{6}$ \\
\hline \multirow[t]{5}{*}{ Triaxiality } & $p$ & 0.73 & 0.73 & 0.72 & 0.74 \\
\hline & $q$ & 0.60 & 0.67 & 0.59 & 0.66 \\
\hline & $\theta$ & $70^{\circ}$ & $48^{\circ}$ & $72^{\circ}$ & $52^{\circ}$ \\
\hline & $\phi$ & $-4.5^{\circ}$ & $-8^{\circ}$ & $-4^{\circ}$ & $-6.5^{\circ}$ \\
\hline & $\xi$ & $14^{\circ}$ & $12^{\circ}$ & $14^{\circ}$ & $16^{\circ}$ \\
\hline \multirow[t]{2}{*}{ Goodness of fit } & d.o.f. & 13199 & 13197 & 13199 & 13197 \\
\hline & $\chi^{2} /$ d.o.f. & 1.42 & 1.37 & 1.51 & 1.49 \\
\hline
\end{tabular}

where $a$ is the core radius. Newberg and Yanny considered (i) a true Hernquist profile, which has $\alpha=1$ and $\delta=3$, and (ii) the pure power law case of arbitrary $\alpha$ with $\delta=0$. We do note that more general profiles were also considered, and the data actually fit a profile very well for intermediate cases with a range of $\alpha$ and $\delta$ such that $\alpha+\delta \approx 4$ (this family of models is also known as $\lambda$ - [47] or $\eta$-models [48]). Table 1 shows the two cases considered by Newberg and Yanny. Two fits with pure Hernquist profile are shown in the table as well as two fits to a pure power law with $\alpha \approx 3$, similar to the power law MACHO and OC1 profiles. The Hernquist profile provides a better fit to the data, but the power law is included for comparison. In total there are 14 parameters to fit, which we now discuss. The parameter $M_{f}$, the peak of the absolute magnitude distribution of the turn-off stars, is fixed to 4.2 in obtaining the other parameters.

3.3.1. Position. In both the true Hernquist and power law models, Newberg and Yanny have examined two cases: (1) the spheroid centre is fixed to coincide with the Galactic Centre but the distance $R_{0}$ from the Sun to the Galactic Centre is allowed to vary, and (2) $R_{0}$ is fixed but the spheroid centre is unconstrained and need not coincide with the Galactic Centre. The distance to the spheroid centre, $R_{0}+\Delta x$, is allowed to vary in both cases. For these two cases, the Hernquist models are denoted by $\mathrm{H} 1$ and $\mathrm{H} 2$ and the power law models by P1 and P2, where index 1 refers to a fixed spheroid centre and 2 refers to an unconstrained spheroid centre. For the unconstrained case, we take $(\Delta x, \Delta y, \Delta z)$ to identify the position of the centre of the spheroid relative to the Galactic Centre in 
galactocentric coordinates. The best fit for the centre of the spheroid is found to lie several kpc from the Galactic Centre in the direction of the $y$ axis.

An important result here is the fact that this best fit position of the centre of the spheroid is away from the line of sight to the LMC, and hence would imply a lower spheroid contribution to the optical depth. In the next section we will see that triaxiality provides an effect in the opposite direction.

3.3.2. Triaxiality. Previous observations and models have included the possibility of a flattened (axial) spheroid. The $F / G$ survey used by Newberg and Yanny, however, is the first direct observation to strongly indicate a triaxial distribution. (A triaxial inner halo has been suggested by Larsen and Humphreys [49] as a possible explanation for an asymmetry they observed in their star counts. A triaxial halo has also been considered to explain indirect observations, such as rotation curves; see, e.g., Blitz and Spergel [50].) The triaxial isodensity contours fall on ellipsoids:

$$
r_{\mathrm{TA}}=\sqrt{x^{2}+\frac{y^{2}}{p^{2}}+\frac{z^{2}}{q^{2}}},
$$

with $p \approx 0.7$ and $q \approx 0.6$. The primary axes are rotated from the galactic coordinates by the angles $(\theta, \phi, \xi) \approx\left(50^{\circ}-70^{\circ},\left(4^{\circ}-8^{\circ}\right), 12^{\circ}-16^{\circ}\right)$ - the major $(x)$ and middle $(y)$ axes lie near the plane of the galaxy, with the major axis at approximately $60^{\circ}$ from our line of sight to the Galactic Centre $((\theta, \phi, \xi)$ describe the orientation of the spheroid axes and specify the following sequence of rotations: by $\theta$ about $\hat{\mathbf{z}}$, then $\phi$ about $\hat{\mathbf{y}}^{\prime}$, and finally $\xi$ about $\left.\hat{\mathbf{x}}^{\prime \prime}\right)$. Figure 1 portrays the triaxial spheroid with a shifted centre.

Note that, with this definition, $r_{\mathrm{TA}}$ does not represent the physical distance to a given point, but is equal to the distance along the major axis to the isodensity contour containing that point (only for the specific case $p=q=1$ are these two distances the same). We will call this parameter $r_{\mathrm{TA}}$ the 'triaxial radius'. Then the local triaxial radius (the distance from the spheroid centre along the major axis to the isodensity contour containing the Sun, i.e., the coordinate equivalent of $R_{0}$ ) now becomes $R_{0, \mathrm{TA}} \approx 11.5 \mathrm{kpc}$ for the fixed position models $\mathrm{H} 1$ and $\mathrm{P} 1$ and $R_{0, \mathrm{TA}} \approx 9.5 \mathrm{kpc}$ for the shifted centre models $\mathrm{H} 2$ and $\mathrm{P} 2$ (see table 2 ). The triaxial radius $R_{0, \mathrm{TA}}$ is smaller in the cases with the shifted centres, even though their physical distance is slightly larger, because the shift places the local position nearer to the major axis. In all cases, the major axis passes somewhat between our local position and the LMC, nearer to the local position. Hence the line of sight to the LMC can be expected to pass through denser portions of the spheroid than in the purely spherical case.

We emphasize here the important effect that the shape of the triaxial spheroid tends to move matter into the line of sight between us and the LMC (as compared to a spherical spheroid), so that the spheroid optical depth would be higher and one would require fewer MACHOs. In section 5 we will see that the two competing effects of (i) position of the centre of the spheroid and (ii) triaxiality of the spheroid tend to cancel.

Henceforth, in order to allow for triaxiality, we replace $r$ with $r_{\mathrm{TA}}$ as defined in equation (6) in the density profiles. 


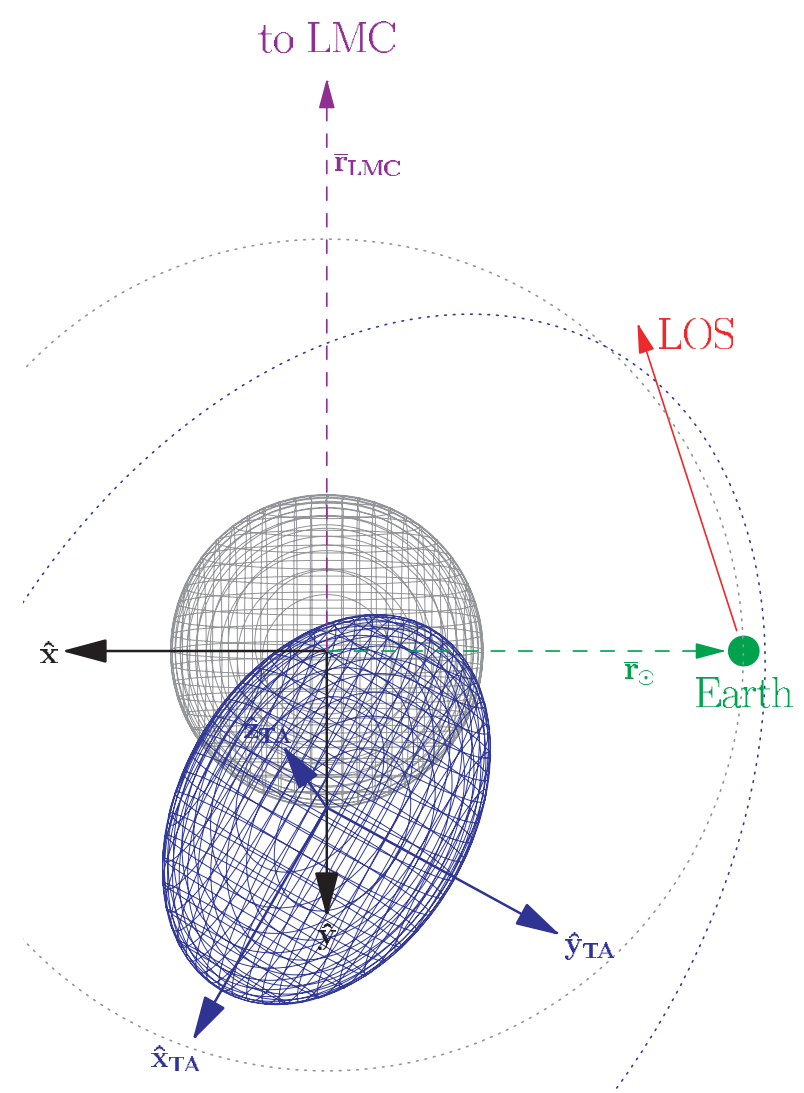

Figure 1. The location and shape of the spheroid found by Newberg and Yanny are shown in galactocentric coordinates (the $+z$ axis is out of the page). The (grey) sphere represents the isodensity contour $3 \mathrm{kpc}$ from the Galactic Centre for a spherical density profile. The (blue) ellipsoid indicates the equivalent triaxial isodensity contour (taking the spheroid mass to be the same as in the spherical case), with a shifted centre; the triaxial axes are also shown. The outer, dotted (grey) circle represents the extent of the spherical contour at $8 \mathrm{kpc}$; the dotted (blue) ellipse is the extent of the equivalent triaxial contour. The line of sight (LOS) to the LMC near Earth passes farther within the triaxial contour than the spherical contour - the densities along the LOS are therefore larger in this region for the triaxial case.

3.3.3. Density normalization. Assuming that the $F / G$ stars track the mass profile of the spheroid, and using equation (5) with the substitution $r \rightarrow r_{\mathrm{TA}}$ (as discussed in the previous section), we can write the density profile of the spheroid as

$$
\rho\left(r_{\mathrm{TA}}\right)=\rho_{\mathrm{S} 0}\left(\frac{R_{0, \mathrm{TA}}}{r_{\mathrm{TA}}}\right)^{\alpha}\left(\frac{a+R_{0, \mathrm{TA}}}{a+r_{\mathrm{TA}}}\right)^{\delta},
$$

where the constant $C$ appropriate to the $F / G$ star density has been replaced by appropriate terms with the local triaxial radius. We still need to determine the overall normalization for the mass density profile for this Newberg/Yanny profile.

The $F / G$ stars in the turnoff phase have masses $\sim 0.85 M_{\odot}$; these stars alone then contribute $\sim 1 \times 10^{-6} M_{\odot} \mathrm{pc}^{-3}$ to the local density. However, main sequence stars, relics, 
Table 2. Several derived parameters for the various models. The local spheroid density is given using both the globular cluster (GC) and mass function (MF) estimates (described in the text). For comparison, the local density used by the MACHO Collaboration is $1.18 \times 10^{-4} M_{\odot} \mathrm{pc}^{-3}$.

\begin{tabular}{llllll}
\hline Model & & $\mathrm{H} 1$ & $\mathrm{H} 2$ & $\mathrm{P} 1$ & $\mathrm{P} 2$ \\
\hline Local coordinate distance & $R_{0, \mathrm{TA}}(\mathrm{kpc})$ & 11.4 & 9.5 & 11.7 & 9.7 \\
Local $F / G$ number density & $n_{F / G, 0}\left(\times 10^{-6} \mathrm{pc}^{-3}\right)$ & 1.12 & 1.11 & 1.35 & 1.47 \\
Local density $(\mathrm{GC})$ & $\rho_{\mathrm{S} 0}\left(\times 10^{-5} M_{\odot} \mathrm{pc}^{-3}\right)$ & 1.1 & 1.1 & 1.4 & 1.5 \\
Local density $(\mathrm{MF})$ & $\rho_{\mathrm{S} 0}\left(\times 10^{-5} M_{\odot} \mathrm{pc}^{-3}\right)$ & 4.5 & 4.4 & 5.4 & 5.9 \\
\hline
\end{tabular}

brown dwarfs and other objects also contribute to the spheroid mass and must be included. We will estimate the total local spheroid density $\rho_{\mathrm{S} 0}$ from the number density profile normalization, $C$ of equation (5), using two different methods.

The first density determination is made by assuming the spheroid population is similar to that of a globular cluster. The Pal 5 globular cluster, with a mass of $4.5-6 \times 10^{3} M_{\odot}[51]$, contains 620 stars with the same colour cuts as the spheroid data set (excluding the $r-i$ cut, which is not significant in this case). Off-field observations predict $\sim 32$ background stars in the Pal 5 field, giving $~ 588$ stars above background. This yields a total stellar mass of approximately $10 M_{\odot}$ per $F / G$ turnoff star. Assuming the spheroid contains a similar stellar population (an assumption which would need to be justified), the spheroid would then have a total local density $\rho_{\mathrm{S} 0} \sim 1 \times 10^{-5} M_{\odot} \mathrm{pc}^{-3}$; values for the specific models are given in table 2 .

Alternatively, we can use the spheroid mass function [1,2], [24]-[27],

$$
\frac{\mathrm{d} n}{\mathrm{~d} M}(M) \propto \begin{cases}M^{-1.1}, & M \lesssim 0.71 M_{\odot} \\ M^{-2.7}, & M \gtrsim 0.71 M_{\odot}\end{cases}
$$

where this particular MF is taken from Gould et al [27] (using the binary corrected form); the general procedure here follows the one of Gould et al, albeit with an independent normalization. The $M^{-1.1}$ power law is thought to be valid for $0.09 M_{\odot}<M<0.71 M_{\odot}$ and the $M^{-2.7}$ power law for $M \gtrsim M_{\odot}$. We have extended the low mass power law to all masses below $0.71 M_{\odot}$. The results are not particularly sensitive to where the power law changes in the range $0.71-1 M_{\odot}$; here, we follow Gould et al and take the change to occur at $0.71 M_{\odot}$. To determine the normalization of the MF, we assume the spheroid stars were predominantly formed at the same time, during the formation of the spheroid $\sim 10^{10}$ years ago; then the stars currently in the turnoff phase (lasting $\sim 3 \times 10^{9}$ years) correspond to a range of masses with width $\Delta \tilde{M} \approx 0.07 M_{\odot}$ centred at $\tilde{M} \approx 0.85 M_{\odot}$. Though not all star formation occurred at the same time, the number of $F / G$ turnoff stars should still give a reasonable estimate for the number of stars in this range, so that

$$
n_{F / G} \approx \frac{1}{2} \frac{\mathrm{d} n}{\mathrm{~d} M}(\tilde{M}) \Delta \tilde{M},
$$

where the extra factor of $1 / 2$ is due to the fact that the selection of $F / G$ stars in the data corresponds to approximately the latter half of the turnoff phase (colour cuts used to obtain this selection are meant to avoid contamination of the sample from other stellar 
populations). Using equation (9) to fix the normalization in equation (8) and noting that progenitors with $M \gtrsim 0.9 M_{\odot}$ have mainly become white dwarfs with $M \approx 0.6 M_{\odot}$, we integrate equation (8),

$$
\rho_{\mathrm{S} 0}=\int \mathrm{d} M M \frac{\mathrm{d} n}{\mathrm{~d} M}
$$

to find the spheroid contains about $40 M_{\odot}$ of mass per $F / G$ turnoff star, or $\rho_{\mathrm{S} 0} \sim$ $5 \times 10^{-5} M_{\odot} \mathrm{pc}^{-3}$. This value varies by $\lesssim 20 \%$ if the MF power law change in equation (8) is taken to occur in the range $[0.71,1.0] M_{\odot}$. Values for the specific models are given in table 2 .

The MF estimate of the local density is $\approx 4$ times larger than the globular cluster estimate, but both are below that determined by most other models, although these estimates are not as rigorous and should be regarded as rough estimates. The exception is the high galactic latitude measurements of Gould et al [27], which provides a very similar estimate of the local density $\left(6.4 \times 10^{-5} M_{\odot} \mathrm{pc}^{-3}\right)$ as our MF estimate (while we use the same MF as Gould et al, we remind the reader that we have independently estimated the normalization of this MF). But, as our estimates are non-rigorous, this similarity may only be a coincidence. A discussion of the local density can be found in section 4 .

We will henceforth use three possible values for the local density:

(i) $\mathrm{MACHO}: \rho_{\mathrm{S} 0}=1.18 \times 10^{-4} M_{\odot} \mathrm{pc}^{-3}$,

(ii) GC: $\rho_{\mathrm{S} 0} \sim 1 \times 10^{-5} M_{\odot} \mathrm{pc}^{-3}$, with more precise values given in table 2 ,

(iii) $\mathrm{MF}: \rho_{\mathrm{S} 0} \sim 5 \times 10^{-5} M_{\odot} \mathrm{pc}^{-3}$, with more precise values given in table 2 .

\section{Importance of various factors for optical depth}

The optical depth results depend on several factors: the triaxiality, the position, the density profile, and the normalization (i.e. total mass or local density) of the spheroid. We discuss each of these.

\subsection{Triaxiality}

A triaxial spheroid can lead to a larger optical depth to the LMC for two reasons: (1) a larger amount of matter along the line of sight and (2) more effective placement of the matter. The line of sight between us and the LMC does not pass much closer to the Galactic Centre than our current position. Hence, for a spherical density distribution, the spheroid density along the line of sight is largest near us and falls off essentially the entire way to the LMC. The models discussed in this paper, however, indicate that the spheroid is triaxial, with the LMC line of sight passing somewhere near the major axis. This means that the line of sight passes through contours of higher density and the total matter along the line of sight is larger than in the spherical case, leading to a larger optical depth.

Not only is the total amount of matter along the line of sight important, but its location along that line is also significant. Lensing objects near the source or observer do not contribute as significantly to the optical depth as lensing objects halfway in between, as is apparent from the $x(1-x)$ factor in the integrand of equation (1). Objects near the observer have small $x$ while objects near the source have small $(1-x)$; the largest 
contribution to the integral is for $x \sim 1 / 2$. This factor has geometrical origins: a lensing object near the source (or observer) must bend the light through a greater angle than one approximately halfway between. Thus a lensing object near the source (or observer) must be much nearer to the line of sight to produce a microlensing event; such an object near the source or observer has a smaller lensing cross-section. Lensing objects approximately halfway along the line of sight, where $x(1-x)$ is maximized, have the largest lensing crosssection and contribute more to the optical depth. For a spherical density distribution, the spheroid density is largest near us $(x \approx 0)$, where the contribution to the optical depth is suppressed due to this geometrical effect. In the triaxial models discussed, the largest densities are not at our location, but farther away where spheroid objects are more likely to produce lensing events. Even if the total amount of matter along the line of sight were the same, the triaxial models in this paper would yield a higher optical depth than spherical ones.

We demonstrate these effects with an example shown in figure 2. For clarity, we examine the optical depth toward a source in the Milky Way nearer than the LMC and take an exaggerated triaxiality $(p=q=0.25)$ in the OC1 model (using a fixed mass normalization). In this figure, we show the spheroid density contours and optical depth toward the source (in units of $10^{-7}$ ) for various spheroid configurations where the major axis is fixed to lie somewhere along the line of sight (LOS). In addition to the optical depth, each panel shows the integral of the density along the LOS $\left(\int \rho\right.$, corresponding to the total amount of intervening matter, in arbitrary units) and the ratio of these two quantities (representing the lensing efficiency). Panel (a) shows the spherical $(p=q=1)$ case. Panel (b) shows a triaxial spheroid with the major axis direction chosen to maximize the amount of matter along the LOS. Note that the LOS passes through much higher density contours than the spherical case, with $\sim 6$ times the mass along the LOS. Most of this additional mass, however, is near to Earth, where $x$ is small, so is not very efficient at producing lensing events. This notwithstanding, the optical depth increases by a factor of five. Panel (c) takes the major axis direction maximizing the lensing efficiency, as determined from the ratio. Note that this occurs when the major axis passes near the midpoint of the LOS, where $x(1-x)$ is maximized. However, the LOS passes through lower density contours than in case (b). With 35\% less intervening mass, the higher efficiency does not lead to a significant increase in the optical depth. Panel (d) takes the major axis direction maximizing the optical depth. This direction is the optimal trade-off between maximizing mass and maximizing efficiency, so falls between cases (b) and (c).

For these two reasons, more mass and better placement, the triaxial versions of nearly all the models examined in this paper lead to larger optical depths than the spherical versions, by roughly $\sim 20-50 \%$ (as shown in the next section, see table 3 ).

\subsection{Position}

An additional consideration taken into account in these models is the centre of the spheroid. Models H2 and P2 have the spheroid location determined from the data, rather than fixed to the Galactic Centre. The centre that best fits the data is actually several kpc from the Galactic Centre, approximately $3 \mathrm{kpc}$ along the $y$ axis in galactocentric coordinates. This direction is away from the LMC line of sight, so these shifted spheroid models lead to lower optical depths. 
Optical depth to the LMC for a triaxial Milky Way spheroid

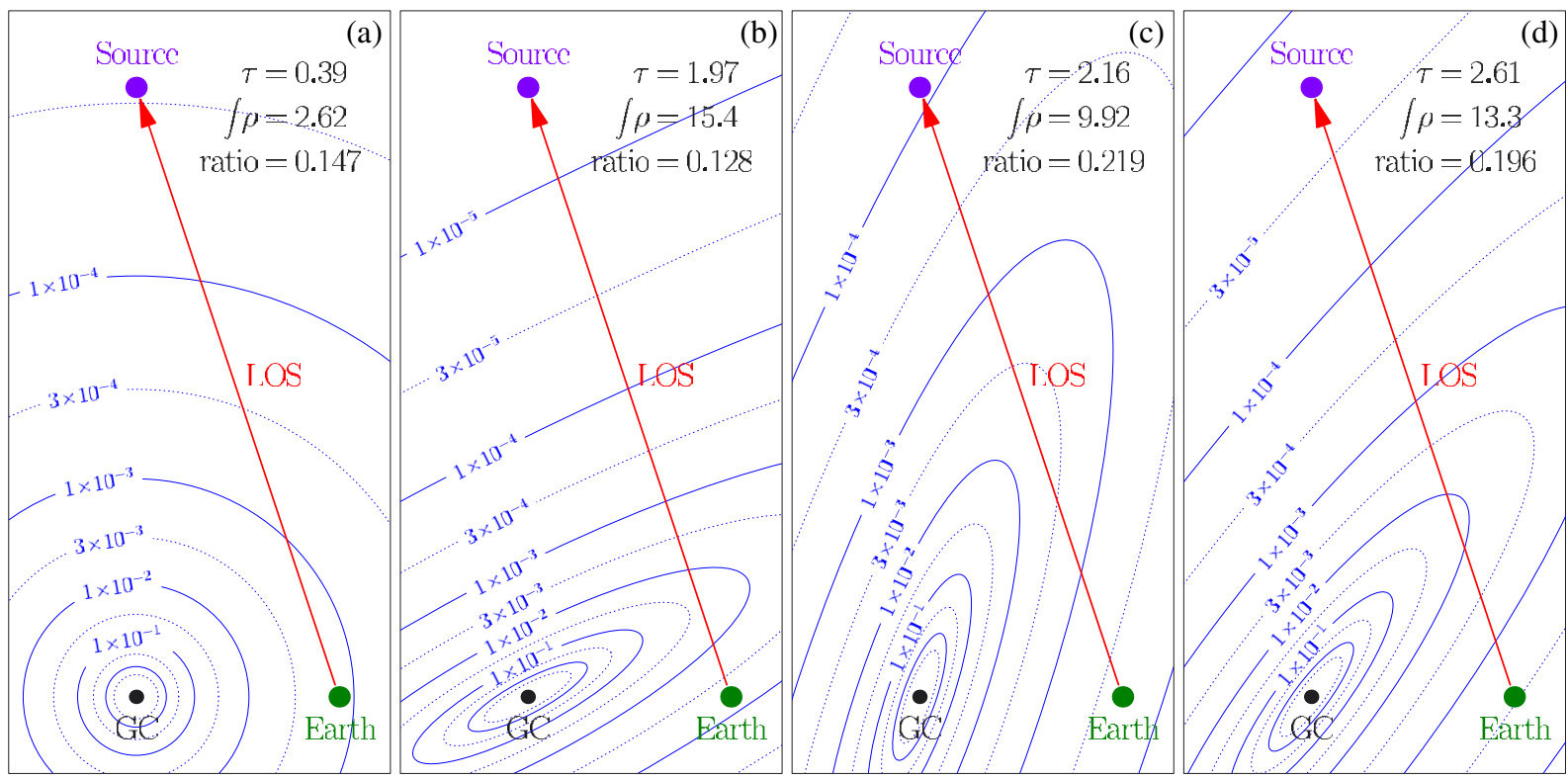

Figure 2. The spheroid density contours and optical depth toward a source in the Milky Way for various spheroid configurations. A nearer source than the LMC and an exaggerated triaxiality $(p=q=0.25)$ have been chosen for clearer visualization; the major axis lies somewhere along the line of sight (LOS). We use the OC1 model with a fixed mass normalization as an illustration. In the upper right hand corner of each panel, we list the optical depth $\tau$ (in units of $\left.10^{-7}\right)$, the integral of the density along the LOS $\left(\int \rho\right.$, corresponding to the total intervening amount of matter, in arbitrary units), and the ratio of these two quantities (representing the lensing efficiency). Panel (a) shows the spherical $(p=q=1)$ case, while panels (b), (c), and (d) show major axis directions that maximize the mass along the LOS, lensing efficiency, and optical depth, respectively.

An off-centred spheroid is not unreasonable from a dynamical standpoint. Some excited states of the galaxy involve weakly damped modes that allow a shifted centre to remain for extended periods of time [52]. Disturbances, including interactions with nearby galaxies (such as the LMC), can pump this excited state back up. However, the size of this offset, $\approx 3 \mathrm{kpc}$, is larger than the offsets typically discussed in the field.

The uncertainty in the position of the spheroid centre is dominated by systematics. Regions of known clumps of stars and contamination are removed when fitting the SDSS data; the remaining regions are assumed to represent a smooth spheroid distribution. However, an unknown clump/stream of stars (perhaps from another, as yet undetected, galaxy being absorbed by the Milky Way) could skew the results and lead to a shift in the fits. Fits would be particularly sensitive to such structure at the South Galactic Cap, due to the limited SDSS data in this region (three stripes). However, the structure would have to be significant in size to produce such a shift in the fit - on the scale of the tidal streams of the Sagittarius dwarf galaxy $[53,54]$ (note all three stripes in the South Galactic Cap contribute to the shift in the fit). In addition, the velocity profiles of stars within these stripes do not give a noticeable indication of any significant structure in the region. 
Table 3. The optical depth due to the Milky Way spheroid (shown in units of $10^{-7}$ ) for the four density profiles described in the text: the upper panel shows results for MACHO, OC1, and power law profiles while the lower panel shows results for Hernquist profiles. Results are presented for both spherical and triaxial spheroids, for the two cases for which the spheroid centre is located at and shifted away from the Galactic Centre. The spherical results are determined by setting $p=1$ and $q=1$, but leaving the remaining parameters as given in table 1 . The local spheroid density $\rho_{\mathrm{S} 0}$ has been determined in three ways (MACHO, GC, and MF) as described in section 3.3.3.

\begin{tabular}{|c|c|c|c|c|}
\hline & \multicolumn{2}{|c|}{ Traditional Galactic Centre } & \multicolumn{2}{|c|}{ Shifted spheroid centre } \\
\hline & Spherical & Triaxial & Spherical & Triaxial \\
\hline $\begin{array}{l}\text { Parameters } \\
\left(R_{0}+\Delta x, \Delta y, \Delta z\right)(\mathrm{kpc}) \\
(\theta, \phi, \xi) \\
(p, q)\end{array}$ & $(1,1)^{(72}$ & $\begin{array}{l}0,0) \\
\left.4^{\circ}, 14^{\circ}\right) \\
(0.72,0.59)\end{array}$ & $\begin{aligned} &(8.2, \\
&\left(52^{\circ},-\right. \\
&(1,1)\end{aligned}$ & $\begin{array}{l}9,0.0) \\
\left.6.5^{\circ}, 16^{\circ}\right) \\
(0.74,0.66)\end{array}$ \\
\hline $\begin{array}{l}\text { Optical depths (in units of } 10^{-7} \text { ) } \\
\text { MACHO }\left(r^{-3.5}\right) \\
\text { OC1 }\left(r^{-3}\right) \\
\text { Power Law }\left(r^{-\alpha}\right) \\
\rho_{\mathrm{S} 0} \text { (MACHO) } \\
\rho_{\mathrm{S} 0}(\mathrm{GC}) \\
\rho_{\mathrm{S} 0}(\mathrm{MF})\end{array}$ & $\begin{array}{l}0.030 \\
0.40 \\
0.037\end{array}$ & $\begin{array}{c}0.044 \\
0.51 \\
(\text { Model P1) } \\
0.052 \\
0.0059 \\
0.024\end{array}$ & $\begin{array}{l}0.021 \\
0.27 \\
0.028\end{array}$ & $\begin{array}{c}0.025 \\
0.46 \\
\text { (Model P2) } \\
0.032 \\
0.0040 \\
0.016\end{array}$ \\
\hline $\begin{array}{l}\text { Parameters } \\
\left(R_{0}+\Delta x, \Delta y, \Delta z\right)(\mathrm{kpc}) \\
(\theta, \phi, \xi) \\
(p, q)\end{array}$ & $(1,1)^{\left(70^{\circ}\right.}$ & $\begin{array}{l}0,0) \\
\left.1.5^{\circ}, 14^{\circ}\right) \\
(0.73,0.60)\end{array}$ & $\begin{array}{r}(8.2, \\
(1,1)^{\left(48^{\circ},\right.}\end{array}$ & $\begin{array}{l}9,0.0) \\
\left.8^{\circ}, 12^{\circ}\right) \\
(0.73,0.67)\end{array}$ \\
\hline $\begin{array}{l}\text { Optical depths (in units of } 10^{-7} \text { ) } \\
\text { Hernquist }\left[r^{-1}(a+r)^{-3}\right] \\
\rho_{\mathrm{S} 0}(\mathrm{MACHO}) \\
\rho_{\mathrm{S} 0}(\mathrm{GC}) \\
\rho_{\mathrm{S} 0}(\mathrm{MF})\end{array}$ & 0.047 & $\begin{array}{l}\text { (Model H1) } \\
0.059 \\
0.0055 \\
0.022\end{array}$ & 0.036 & $\begin{array}{l}\text { (Model H2) } \\
\quad 0.035 \\
0.0033 \\
0.013\end{array}$ \\
\hline
\end{tabular}

It has been brought to our attention by Gould [55] that the dynamics of a shifted spheroid might lead to a net motion of the local spheroid population with respect to the galactic rest frame, at odds with current observations $[39,56]$. The shift (as well as a triaxial shape) might also lead to non-trivial cross terms in the velocity dispersion covariance matrix. These considerations are not examined in this paper, but will be investigated in the future.

\subsection{Density profile}

The choice of density profile is also an important factor in the optical depth prediction. The profiles that fall off more slowly with distance from the spheroid centre have more mass along the line of sight and hence a higher optical depth. For example, the Hernquist 
fit profile $\left(r^{-1}(a+r)^{-3}\right)$ falls as $r^{-1}$ for a significant portion of the LMC line of sight and it does not reach the more rapid $r^{-4}$ drop until $r \gg a \approx 15 \mathrm{kpc}$. The MACHO profile $\left(r^{-3.5}\right)$ and power law $F / G$ fit profile $\left(r^{-\alpha}\right.$, where $\left.\alpha \approx 3\right)$, on the other hand, fall rapidly at all distances. For a fixed local spheroid density, the Hernquist profile will yield higher densities at farther distances than the other profiles and result in a larger optical depth.

\subsection{Density normalization}

The factor that has the strongest effect on the optical depth is the normalization of the spheroid density. We use four normalization choices. The lightest local density is due to the GC and MF normalizations arising from the $F / G$ turnoff star counts (Newberg/Yanny data; see table 2), the local density from the MACHO Collaboration is up to a factor of ten higher, and the OC1 normalization is by far the largest.

Throughout the paper, we use the local spheroid density and density profile normalization interchangeably. Indeed, the normalization of the density profiles (equations (2), (4), and (7)) is explicitly defined in terms of the local density $\rho_{\mathrm{S} 0}$. Implicitly assumed in these equations, however, is that the spheroid is composed of a single, smooth component, in which case the $\rho_{\mathrm{S} 0}$ parameter used to define the normalization in these equations is, in fact, the actual local density of the spheroid. If the spheroid is more complex, such as containing structure or multiple components, the term 'local spheroid density' becomes ambiguous: the local density used in the density profile equations represents only the smooth component described by this profile and does not include additional structure or spheroid components, while the 'actual' local density, measured by local star counts, would include these additional components. It is important to note that the $F / G$ models discussed here have their density profile normalizations directly fixed by the observations; the local densities discussed for these models are deduced from this overall normalization and represent only the smooth spheroid component (the local density is not measured). The MACHO model takes the observed (actual) local density equation (3) and assumes it to be entirely representative of the smooth spheroid given by equation (2).

In the remainder of this section, we discuss the ways in which the $F / G$ turnoff star normalizations ( $\mathrm{GC}$ and $\mathrm{MF}$ ) might be made compatible with the MACHO Collaboration normalization.

Could the $F / G$ normalization estimates indicate a lighter spheroid than previously believed? The high galactic latitude star counts of [27] would also suggest this, with a predicted local density, $6.4 \times 10^{-5} M_{\odot} \mathrm{pc}^{-3}$, two to three times smaller than the estimates from local high velocity star observations. In order for local high velocity stars to give an inaccurate indication of the spheroid normalization, leading to a heavier spheroid prediction, the local density must be higher than the smooth background distribution due to the presence of a 'lump', stream, or additional spheroid component. Indeed, we know that such streams exist - the leading edge of the Sagittarius dwarf galaxy passes very near to the solar neighbourhood and could contribute a set of high proper motion stars [53]. If this stream was, however, the dominant component of the local high velocity stars, it would likely have been noticed in a kinematic analysis of these stars: their motions would be highly correlated (see, e.g. Helmi et al [57], who find what appears to be a local stream, but not of a significant density). Multiple streams might mask this signature by 
making the velocities appear more isotropic, leading to a local density determination that overestimates that of the spheroid. Gould [56], though, argues from a statistical analysis of local halo stars that the stellar halo is unlikely to be dominated by streams in our neighbourhood.

Another explanation has been proposed by Sommer-Larsen and Zhen [58] and studied by others $[34,59]$ : a two component spheroid containing the traditional approximately spherical distribution as well as an additional highly flattened component (not to be confused with the disk, as this component has no net rotation). This model leads to approximately equal contributions to the local spheroid density from the two components; however, the flattened component does not contribute to non-local star counts such as that of Gould et al and the $F / G$ turnoff stars used in this paper. In this case, the local spheroid density estimates in Gould et al and those based on $F / G$ turnoff stars (see table 2) represent only the extended spheroid contribution and not the flattened component contribution. Local star counts, which contain both components, would naturally yield a higher estimate of the local density, by approximately a factor of two, and there would be no discrepancy between these two types of observations.

Alternatively, one can consider the possibility that our mechanisms for determining the $F / G$ normalizations are yielding underestimates. The GC estimate is made by assuming the spheroid stellar population is similar to that of a globular cluster, yielding similar mass-to-light ratios. However, if low mass stars evaporate from globular clusters, the cluster mass-to-light ratio would be lower than that of the spheroid and our GC normalization estimate would then likewise be low [27].

The MF $F / G$ normalization estimate was determined by using the spheroid mass function from Gould et al [27]. The Gould et al MF, given by equation (8), was determined by examining faint (hence, distant spheroidal) main sequence stars at high galactic latitudes, so should probably not have been contaminated by a local bump or a second spheroid component, as discussed above, and should be reasonably valid for the $F / G$ models (also non-local star counts). However, inferring an MF from such star counts is no simple matter, and some uncertainties exist. A study of spheroid stellar populations by Gould et al [39] suggests there may be more low mass stars than accounted for by the Gould et al $\mathrm{MF}^{4}$. In this case, our $F / G$ normalization estimate would be low. Both MFs are in agreement for the number of higher mass spheroid stars and extrapolation to turnoff star masses predicts a number of turnoff stars consistent with that observed by the $F / G$ survey. Thus, the $F / G$ survey does not conflict with either of these MFs. Since the disagreement between these MFs is for low mass stars and the $F / G$ survey only includes (high mass) turnoff stars, the $F / G$ models do not give us an indication of which MF is correct $^{5}$.

We wish to emphasize that our two estimation methods provide only rough estimates: the factor of four difference between them should be an indication of this. While these estimates might point to a lighter spheroid mass than that predicted by local star counts (and used by MACHO), given the uncertainties in our derivations and the issues discussed above, our estimates should not be considered to be incompatible with those of the local

4 The Gould paper is based upon kinematically selected local stars and could, in principle, be measuring a different population of stars (including, e.g., a second spheroid component as discussed previously) than the non-local observations of Gould et al.

${ }^{5}$ We thank the referee for bringing this issue to our attention. 
star counts. The $F / G$ estimate using the $\mathrm{MF}\left(\rho_{\mathrm{S} 0} \sim 5 \times 10^{-5} M_{\odot} \mathrm{pc}^{-3}\right)$ is only two to three times smaller than the MACHO local density $\left(\rho_{\mathrm{S} 0}=1.18 \times 10^{-4} M_{\odot} \mathrm{pc}^{-3}\right)$; it is certainly possible that this simply arises due to approximations made in the estimation process (yielding underestimates at this level). As such, we draw no conclusions as to whether the MACHO and $F / G$ estimated local densities are incompatible.

\section{Results}

We have examined four spheroid models:

(i) a profile $\rho(r) \propto r^{-3.5}$ used by the MACHO collaboration and based upon local high velocity star counts and high galactic latitude star counts, see equation (2);

(ii) a profile $\rho(r) \propto r^{-3}$ referred to as $\mathrm{OC} 1$ and obtained via galactic dynamics, see equation (4);

(iii) a new model, a true Hernquist profile of equation (7), with $\alpha=1$ and $\delta=3$, obtained by fits to spheroid $F / G$ turnoff stars; and

(iv) a power law profile of equation $(7), \delta=0$, also obtained by fits to spheroid $F / G$ turnoff stars.

For all four of the models, we have allowed for the possibility of triaxiality by replacing $r$ with $r_{\mathrm{TA}}$ in the profiles, defined in equation (6). We have also allowed the spheroid centre to move away from the Galactic Centre. Our results are shown in table 3. We will now discuss each of the models in turn.

\subsection{MACHO model}

Normalization. Since the density profile equation (2) in the MACHO model is based partly on local star counts, which presumably gives a reliable indication of the local spheroid density regardless of the spheroid model, we use the local spheroid density given in equation (3) as the local density in the triaxial case as well.

Triaxiality and position. In table 1, we obtained the best fits to the Newberg/Yanny data for the triaxility and central spheroid position for arbitrary power law density profiles (models P1 and P2). Though the best fit power law index in the table $\left(\rho \propto r^{-\alpha}\right)$ was closer to $\alpha=3.0$ rather than the $\alpha=3.5$ of the MACHO profile, still we feel confident that we can use the results of models P1 and P2 in table 1 as a good estimate of triaxiality and position for the MACHO case as well.

Results for spheroid contribution to optical depth. As shown in table 3, triaxiality boosts the spheroid optical depth of this profile by $(25-50) \%$ for either choice of the spheroid centre. However, shifting the spheroid centre suppresses the optical depth as the densest portions of the spheroid are moved away from the LMC line of sight. In addition, the highest densities along the line of sight are closer to the local position, where the microlensing cross-section is smaller (note that this corresponds to $x \approx 0$ in equation (1), so the integrand is small even where the density is large). The combination of these two modifications (spheroid shape and position) results in a decrease of the spheroid contribution to the optical depth from $0.030 \times 10^{-7}$ in the traditional model to $0.025 \times 10^{-7}$, a drop of $\sim 20 \%$. Since the spheroid contribution to the overall optical depth 
was only a few per cent in the spherical MACHO case, this change is unimportant to the estimate of the number of MACHOs in the Halo of our Galaxy.

\subsection{Dynamical models (OC1)}

Normalization. Dynamical models are based upon rotation curves and are sensitive to the spheroid mass. Thus for the OC1 profile (equation (4)), we fix the spheroid mass (rather than the local density) when modifying the model for a triaxial shape.

Triaxiality and position. Again, the spheroid position and triaxiality are taken from models $\mathrm{P} 1$ and $\mathrm{P} 2$ in table 1.

Results for spheroid contribution to optical depth. Triaxiality gives a (25-70)\% boost in the optical depth, but again the shifting of the spheroid suppresses the optical depth, resulting in an overall increase from $0.40 \times 10^{-7}$ to $0.46 \times 10^{-7}$ when both effects are taken into account. The much heavier spheroid predicted by this model results in a sizable contribution to the optical depth in all cases. For the original spherical OC1 spheroid, $50 \%$ of the excess optical depth to the LMC was due to the spheroid, so that the maximum MACHO contribution to the dark matter of the Milky Way Halo was roughly 10\%. With the triaxiality taken into account, the new number would be roughly $8 \%$. However, since the errors on the observed optical depth from the MACHO experiment to $1 \sigma$ are as high as 30\%, unfortunately the variation in the optical depth between the spherical and triaxial cases is smaller that the uncertainties in the observed optical depth.

\subsection{New spheroid model: Hernquist profile with Newberg/Yanny fits}

Our third set of models uses the Hernquist profile with fits to the Newberg/Yanny data. Model H1 has a triaxial spheroid with the spheroid centred at the traditional Galactic Centre. Model H2 has a triaxial spheroid with a shifted centre (not at the Galactic Centre). For both $\mathrm{H} 1$ and $\mathrm{H} 2$ we take the values of core radius obtained in table 1 from fits to the Newberg/Yanny data.

Normalization. We use three different normalizations for the density profiles in equation (7). First, we assume the MACHO value for the local density given in equation (3). Second, we use the normalization from the globular cluster comparison (GC). Third, we use the normalization obtained using the spheroid mass function (MF). These latter two cases, discussed previously in section 3.3.3, do not apply to the spherical models; hence, no results for spherical models with GC or MF normalization are presented.

Triaxiality and position. The triaxiality and position of the spheroid centre for models $\mathrm{H} 1$ and $\mathrm{H} 2$ can be found in table 1.

Results. With the local density fixed to the MACHO value (MACHO normalization), the Hernquist profile optical depth increases with triaxiality when the spheroid is fixed to the Galactic Centre (model H1). The contribution to the optical depth is then still only $\sim 5 \%$ of the observed value, leading to at most one or two of the observed microlensing events. However, when the spheroid is shifted as well (model H2), the overall optical depth decreases. This effect is partly due to the fact that, in model H2, our local position is so near the major axis that no dense region 'appears' in the LMC line of sight when going from a spherical to triaxial shape. In fact, our local position is close to the densest portion of the spheroid, so that with a fixed local density the overall mass of the spheroid 
decreases. If we use the GC or MF normalizations for the spheroid density profile, the optical depth becomes so small as to be irrelevant.

\subsection{Power law model fit to Newberg/Yanny data}

Our fourth set of models uses a power law profile with fits to the Newberg/Yanny data. Model P1 has a triaxial spheroid with spheroid centred at the traditional Galactic Centre. Model P2 has a triaxial spheroid with a shifted centre (not at the Galactic Centre). We use the same three normalizations for the density profiles as in the third set of models: MACHO, GC, and MF. The triaxiality and position of the spheroid centre for models P1 and $\mathrm{P} 2$ can be found in table 1.

Results. Our results for the fourth set of models depend on the choice of normalization of the density profile. Using the MACHO normalization for models P1 and P2 with triaxiality, we obtain very similar results to those found with the MACHO profile. This similarity is expected since the MACHO profile is also a power law. The differences stem from the best fit power $\rho \propto r^{-\alpha}$, where $\alpha=3.0$ for P1 and 2.9 for P2, as opposed to $\alpha=-3.5$ assumed by the MACHO group. Because the power law drops a bit more slowly, the fits yield $\sim 25 \%$ larger optical depths. The shifted spheroid centre again suppresses the optical depth, yielding a net decrease of $\sim 10 \%$.

Results are also presented in table 3 for the two other normalizations of the density profile, GC and MF. As mentioned above, these do not apply to the spherical case and hence results are only presented for the triaxial case. We find that the optical depth is $\sim 30 \%$ lower when the spheroid centre is shifted than when it is lined up with the Galactic Centre. For both GC and MF normalizations, the optical depth is too small to be of any significance, $<2 \%$ of the excess MACHO optical depth.

\section{Discussion}

Our results of the previous section depend on the triaxiality of the spheroid, the location of its centre, the density profile, and the normalization. While the importance of these elements has been emphasized previously, here we demonstrate explicitly the dependence of our results on these various factors.

\subsection{Triaxiality}

Due to more mass and better placement, the triaxial versions of nearly all the models examined in this paper lead to larger optical depths than the spherical versions, with increases of $\sim 20-50 \%$. The exception is model $\mathrm{H} 2$ when the local density is fixed. In this model, the major axis is closer to us than any of the other models; this can be seen by noting that the local triaxial radius, $R_{0, \mathrm{TA}}=9.5 \mathrm{kpc}$ (see equation $(6)$ ), is very close to the physical distance, $8.8 \mathrm{kpc}$. Then triaxiality boosts the spheroid density only near us, but points farther away along the line of sight will fall on lower density contours than they would in spherical models; compare figures 2(a) and (b) in our previous example. Triaxiality does not as significantly increase the optical depth in this case (the fixed normalization also plays a part, as mentioned previously).

Clearly, the direction of the major axis matters. In fact, the observed direction in most of the models is rather fortunate as far as optimizing the optical depth. The optical 
depth is maximized for a major axis passing through the line of sight. While the observed direction does not exactly do this, it passes closer to the line of sight than most other directions. In addition, the optical depth is maximized for a major axis near to us (but not too near); the observed direction is reasonably optimal in this regard.

\subsection{Position}

The location of the spheroid centre also affects our results. Models H2 and P2 have the spheroid location determined from the data, rather than fixed to the Galactic Centre. The centre that best fits the data is actually several kpc from the Galactic Centre, approximately $3 \mathrm{kpc}$ along the $y$ axis in galactocentric coordinates. This direction is away from the LMC line of sight, so these shifted spheroid models lead to lower optical depths, by $\sim 25-40 \%$ for a fixed local density, as shown in the previous section. This effect generally goes in the opposite direction to that of triaxiality; the combination of a shifted centre and triaxial shape leads only to a modest increase $(\lesssim 10 \%)$ or decrease $(\lesssim 25 \%)$ in the optical depth for the density profiles considered.

\subsection{Density profile}

Our results for the optical depth also depend on the choice of density profile. To illustrate the impact of the density profile, let us compare the MACHO profile $\left(r^{-3.5}\right)$, power law $F / G$ fit profile $\left(r^{-\alpha}\right)$, and Hernquist fit profile $\left(r^{-1}(a+r)^{-3}\right)$ for the case where the local density is fixed to the value used by the MACHO Collaboration. The MACHO profile yields the smallest optical depth in this case, while the Hernquist profile yields the largest. The reason is the more rapid $r^{-3.5}$ drop in density with the MACHO profile; for a significant portion of the LMC line of sight, the Hernquist profile falls as $r^{-1}$ and it does not reach the more rapid $r^{-4}$ drop until $r \gg a \approx 15 \mathrm{kpc}$. The Hernquist profile, then, results in higher densities at $r>R_{0}$ (distance from us to Galactic Centre) and a higher optical depth. In section 5 we saw that, combined with triaxiality, the prediction for the spheroid optical depth nearly doubles from the original MACHO profile $0.030 \times 10^{-7}$ to the Hernquist profile $0.059 \times 10^{-7}$ (including shifting of the spheroid centre results in only a modest $16 \%$ net increase to $0.035 \times 10^{-7}$ ). The Hernquist profile provides a better fit to the data than a power law, with a reduced $\chi^{2}$ of 1.42 and 1.37 for models $\mathrm{H} 1$ and $\mathrm{H} 2$, respectively, versus 1.51 and 1.49 for models $\mathrm{P} 1$ and $\mathrm{P} 2$, respectively.

\subsection{Normalization}

By far the largest concern in determining if the spheroid can account for the MACHO observed optical depth is the normalization of the models, corresponding to the spheroid mass or local density. The MACHO Collaboration used a value of $1.18 \times 10^{-4} M_{\odot} \mathrm{pc}^{-3}$ for the local density, determined from local (high velocity) and non-local (high galactic latitude) star counts. Since the local measurements presumably fix the local density regardless of the spheroid model, we have examined the optical depth of our power law (P1 and P2) and Hernquist (H1 and H2) profiles using this local density to fix the normalization. In these cases, the optical depth from the spheroid comprises only a relatively small portion of the excess observed value. While model H1 demonstrates that an alternative profile with a triaxial shape can give a relatively large increase over 
the traditional spherical power law profiles, the contribution to the optical depth of $0.059 \times 10^{-7}$ in this case is still only $\sim 7 \%$ of the excess observed value, contributing at most one or two of the observed events.

For the fits to the $F / G$ star surveys (normalizations GC and MF), we also estimated the spheroid density profile normalizations directly from the fit normalizations. The two different estimation techniques both predict a local density up to an order of magnitude smaller than that used by the MACHO Collaboration. For these estimates, the spheroid optical depth is truly insignificant, $\lesssim 3 \%$ of the excess observed value. However, we would like to stress that these were only quick, non-rigorous estimates and should not be regarded as highly accurate.

The only model, then, with optical depths of a significant size is OC1, due to its much heavier spheroid. Even the original model predicts $0.40 \times 10^{-7}$ for the optical depth, $\sim 50 \%$ of the excess observed value. Triaxiality and spheroid position vary this number by as much as 30\% (note that the spheroid mass, not the local density, is fixed for this model). OC1 with a triaxial shape would account for $\sim 65 \%$ of the excess observed value. Triaxiality can lead to a significant increase in the optical depth, but only if the spheroid is heavy and the optical depth is already significant.

We would like to remind the reader that OC1 and other dynamical models with heavy spheroids are based upon rotation curves and other measurements of the inner galaxy. As such, they may provide accurate models of the inner portion of the spheroid, but are not necessarily accurate at the larger radii $\left(r \gtrsim R_{0}\right)$ relevant to the LMC optical depth (note that the LMC line of sight does not pass much closer than $R_{0}$ to the Galactic Centre). The (local and non-local) star counts forming the basis of the MACHO local density are taken at $r \sim 5-20 \mathrm{kpc}$ and are probably more reliable for describing the relevant regions of the galaxy. In addition, while the $F / G$ normalization estimates may not be highly accurate, they would need to be off by almost two orders of magnitude to be compatible with the OC1 model - it is not obvious how these estimates could be off to such a degree.

\section{Summary}

We have investigated the effects of triaxiality of the spheroid, as discovered by Newberg and Yanny, on the optical depth to the LMC, which is used to ascertain the number of MACHOs in our Halo. We showed that there are two competing effects. First, the triaxiality of the fit allows for a larger spheroid mass along the line of sight between us and the LMC; the placement of the mass is also optimal for producing microlensing. This effect leads to a larger optical depth due to the Milky Way spheroid and would imply the existence of fewer MACHOs to fit the data. However, allowing the centre of the spheroid to move (rather than fixing it to coincide with the Galactic Centre) leads to an effect that goes in the other direction: the best fit to the spheroid centre is away from the line of sight to the LMC. As a consequence, these two effects tend to cancel so that the change in optical depth due to the Newberg/Yanny triaxial halo is smaller than the errors in the measurement.

We considered four spheroid models corresponding to four different density profiles. Within these models, we allowed for four different sets of normalization, with different corresponding local densities, and obtained the resulting optical depth. 
Prior to this work there were two widely diverging results for the spheroid optical depth: those from the MACHO experiment and the OC1 model discussed in the text. The reason for the discrepancy is primarily due to a difference in normalization for the local density. Compared to the standard spheroid optical depth obtained by the MACHO experiment, if we continue to use the MACHO normalization and local density, we find that the increase due to triaxiality is at most a factor of two: the spheroid optical depth may increase from one-40th of the total optical depth toward the LMC to one-20th of it. Hence one or two of the observed events may be due to spheroid microlensing. If one considers the alternate heavy OC1 model, triaxiality changes the spheroid contribution from one-half the excess optical depth to two-thirds (within $\sim 1 \sigma$ of the experimental excess). A third normalization for the local density derived from $F / G$ star counts in the Newberg/Yanny data appears to be more consistent with a lighter spheroid and predicts only negligible contributions to the optical depth. For the four spheroid models we have considered, the MACHO contribution to the dark matter in the Galactic Halo is then in the (8-20)\% range if all excess lensing events are assumed to be due to MACHOs, where the maximum is due to the original MACHO Collaboration results and the minimum is consistent with $0 \%$ at the $1 \sigma$ error level in the data.

\section{Acknowledgments}

CS thanks W Sutherland, M Weinberg and A Font for helpful conversations. CS and HJN thank A Gould for comments on the initial draft of this paper. CS and KF acknowledge the support of the DOE and the Michigan Center for Theoretical Physics via the University of Michigan. PG's work was partially supported by NSF grant PHY-0456825. HJN acknowledges funding from NSF grant AST 03-07571.

\section{References}

[1] Graff D S and Freese K, 1996 Astrophys. J. 467 L65 [SPIRES] [astro-ph/9602051]

[2] Graff D S and Freese K, 1996 Astrophys. J. 456 L49 [SPIRES] [astro-ph/9507097]

[3] Freese K, Fields B and Graff D, 2000 Preprint astro-ph/0007444

[4] Alcock C et al (the MACHO Collaboration), 2000 Astrophys. J. 542281 [SPIRES] [astro-ph/0001272]

[5] Lasserre T (EROS Collaboration), 2000 Astron. Astrophys. 355 L39 [SPIRES] [astro-ph/0002253]

[6] Afonso C et al (EROS Collaboration), 2003 Astron. Astrophys. 400951 [SPIRES] [astro-ph/0212176]

[7] Fields B D, Freese K and Graff D S, 2000 Astrophys. J. 534265 [SPIRES] [astro-ph/9904291]

[8] Graff D S, Freese K, Walker T P and Pinsonneault M H, 1999 Astrophys. J. 523 L77 [SPIRES] [astro-ph/9903181]

[9] Fields B D, Freese K and Graff D S, 1998 New Astron. 3347 [astro-ph/9804232]

[10] Paczyński B, 1986 Astrophys. J. 3041 [SPIRES]

[11] Udalski A et al, 1994 Acta Astron. 44165 [astro-ph/9407014]

[12] Udalski A, Zebrun K, Szymanski M, Kubiak M, Pietrzynski G, Soszynski I and Wozniak P, 2000 Acta Astron. 501 [astro-ph/0002418]

[13] Ansari R et al, 1997 Astron. Astrophys. 324843 [SPIRES] [astro-ph/9607040]

[14] Calchi Novati S et al, 2002 Astron. Astrophys. 381848 [SPIRES] [astro-ph/0110706]

[15] Sumi T and Kan-ya Y, 2002 Mon. Not. R. Astron. Soc. 3371017 [astro-ph/0208399]

[16] Sumi T et al, 2003 Astrophys. J. 591204 [SPIRES] [astro-ph/0207604]

[17] de Jong J T A et al (the MEGA Collaboration), 2004 Astron. Astrophys. 417461 [SPIRES] [astro-ph/0307072]

[18] Baltz E A, Lauer T R, Zurek D R, Gondolo P, Shara M M, Silk J and Zepf S E, 2004 Astrophys. J. 610691 [SPIRES] [astro-ph/0310845]

[19] Giudice G F, Mollerach S and Roulet E, 1994 Phys. Rev. D 502406 [SPIRES] [astro-ph/9312047]

[20] Newberg H J and Yanny B, 2005 ASP Conf. Ser. 338: Astrometry in the Age of the Next Generation of Large Telescopes 338210 [astro-ph/0502386] 
[21] Newberg H J and Yanny B, 2005 Proc. Workshop on Physics at the End of the Galactic Cosmic Ray Spectrum (April 2005) at press [astro-ph/0507671]

[22] Paczyński B, 1996 Ann. Rev. Astron. Astrophys. 34419 [SPIRES] [astro-ph/9604011]

[23] Roulet E and Mollerach S, 1997 Phys. Rep. 27967 [SPIRES] [astro-ph/9603119]

[24] Scalo J M, 1986 Fundam. Cosm. Phys. 111

[25] Gould A, Bahcall J N and Flynn C, 1997 Astrophys. J. 482913 [SPIRES] [astro-ph/9611157]

[26] Chabrier G, 2003 Publ. Astron. Soc. Pac. 115763 [astro-ph/0304382]

[27] Gould A, Flynn C and Bahcall J N, 1998 Astrophys. J. 503798 [SPIRES] [astro-ph/9711263]

[28] Schmidt M, 1975 Astrophys. J. 20222 [SPIRES]

[29] Bahcall J N and Casertano S, 1986 Astrophys. J. 308347 [SPIRES]

[30] Dahn C C, Liebert J, Harris H C and Guetter H H, 1995 Proc. ESO Workshop, The Bottom of the Main Sequence - and Beyond (Aug. 1994) ed C G Tinney (Berlin: Springer) p 239

[31] Gizis J E and Reid I N, 1999 Astron. J. 117508 [SPIRES] [astro-ph/9810071]

[32] Cooke J A and Reid I N, 2000 Mon. Not. R. Astron. Soc. 3181206

[33] Norris J, Bessell M S and Pickles A J, 1985 Astrophys. J. Suppl. 58463

[34] Chiba M and Beers T C, 2000 Astron. J. 1192843 [SPIRES] [astro-ph/0003087]

[35] Bahcall J N and Soneira R M, 1980 Astrophys. J. Suppl. 4473

[36] Digby A P, Hambly N C, Cooke J A, Reid I N and Cannon R D, 2003 Mon. Not. R. Astron. Soc. 344583 [astro-ph/0304056]

[37] Lemon D J, Wyse R F G, Liske J, Driver S P and Horne K, 2004 Mon. Not. R. Astron. Soc. 3471043 [astro-ph/0308200]

[38] Bahcall J N, Schmidt M and Soneira R M, 1983 Astrophys. J. 265730 [SPIRES]

[39] Gould A, 2003 Astrophys. J. 583765 [SPIRES] [astro-ph/0208004]

Gould A, 2004 Astrophys. J. $\mathbf{6 0 7} 653$ (erratum)

[40] Sutherland W, 2005 private communication

[41] Caldwell J A R and Ostriker J P, 1981 Astrophys. J. 25161 [SPIRES]

[42] Ostriker J P and Caldwell J A R, 1983 Kinematics, Dynamics and Structure of the Milky Way ed Shuter W L H (Dordrecht: Reidel) pp 249-57

[43] Rohlfs K and Kreitschmann J, 1988 Astron. Astrophys. 20151 [SPIRES]

[44] York D G et al (SDSS Collaboration), 2000 Astron. J. 1201579 [SPIRES] [astro-ph/0006396]

[45] Abazajian K et al (SDSS Collaboration), 2005 Astron. J. 1291755 [SPIRES] [astro-ph/0410239]

[46] Hernquist L, 1990 Astrophys. J. 356359 [SPIRES]

[47] Dehnen W, 1993 Mon. Not. R. Astron. Soc. 265250

[48] Tremaine S et al, 1994 Astron. J. 107634 [SPIRES] [astro-ph/9309044]

[49] Larsen J A and Humphreys R M, 1996 Astrophys. J. 468 L99 [SPIRES]

[50] Blitz L and Spergel D N, 1991 Astrophys. J. 370205 [SPIRES]

[51] Odenkirchen M, Grebel E K, Dehnen W, Rix H-W and Cudworth K M, 2002 Astron. J. 1241497 [SPIRES] [astro-ph/0206276]

[52] Weinberg M, 2005 private communication

[53] Majewski S R, Skrutskie M F, Weinberg M D and Ostheimer J C, 2003 Astrophys. J. 5991082 [SPIRES] [astro-ph/0304198]

[54] Newberg H J et al, 2003 Astrophys. J. 596 L191 [SPIRES] [astro-ph/0309162]

[55] Gould A, 2005 private communication

[56] Gould A, 2003 Astrophys. J. 592 L63 [SPIRES] [astro-ph/0304267]

[57] Helmi A, White S D M, de Zeeuw P T and Zhao H-S, 1999 Nature 40253 [SPIRES] [astro-ph/9911041]

[58] Sommer-Larsen J and Zhen C, 1990 Mon. Not. R. Astron. Soc. 24210

[59] Siegel M H, Majewski S R, Reid I N and Thompson I B, 2002 Astrophys. J. 578151 [SPIRES] [astro-ph/0206323] 